Though there is little new conceptually and the result is expected, this work is a considerable achievement. It should be applicable to other bacterial genes, and the availability of pure genes should help in the solution of some important remaining problems in the control of gene expression. More generally, the work has again raised fears that biologists may come to abuse all this knowledge-for instance, by producing highly virulent infections for which there is no cure or by tampering with man's genetic endowment. In fact, the Harvard work adds little to already existing worries. The phenomenon of linked resistance against several antibiotics in pathogenic bacteria, now quite common in many countries, causes more trouble in practice than is likely to be achieved using purified genes even in the most diabolical hands. The Harvard team used the most favourable system now known. Much developmental work is required before isolation of a mammalian gene becomes possible, and more again before the isolated gene can be successfully introduced into the genome of a host cell. If this could be achieved, especially in germ cells, genetic deficiencies could be made up, so curing the corresponding hereditary disease. This is a long-term aim which we should all regard as laudable. As far as danger is concerned, the risks of producing genetic damage by radiation or radiomimetic chemicals seem at present far greater than those likely to be caused by abuse of genetic engineering, as the manipulation of genetic material has come to be termed. This discovery is a scientific achievement that need not carry with it any pangs of doubt or regret.

\section{The Experience of Time}

How is it that we, experience the passage of time? We are aware of sound, touch, and colours through sense organs. Could it be that we perceive the flow and duration of time through some time-sense organ within the brain? We nowadays read of "biological clocks," and the pulse, the breathing, the electroencephalographic alpha rhythm, or one of our many diurnal rhythms could perhaps provide a timebase which our time-organ could calibrate against our wristwatches.

In seeking answers to these questions psychologists have studied the perception of time. Among them was H. Hoagland, ${ }^{1}$ whose febrile wife helped in his experiments. When she attempted to count at the rate of one number per second she went faster when her temperature was higher. He related the $\log$ of the counting speed to her absolute temperature and proposed a chemical clock which could make more time seem to pass during pyrexia. Later experiments conducted on divers, whose body temperature varied with immersion in cold or warm sea water, confirmed that the rate of counting was correlated with temperature. ${ }^{2}$

The psychedelic drugs lysergic acid diethylamide (L.S.D. $)^{3}$ and psilocybin ${ }^{5}$ also alter perception of time, so that a short period of clock time seems of much longer duration both subjectively and on testing. Dexamphetamine too has been found ${ }^{6}$ to cause a decrease in the clock-measured value of the apparent second: that is to say, like fever, L.S.D., and psilocybin, it makes the passing time seem long. On the other hand quinalbarbitone $200 \mathrm{mg}$. had the opposite effect and made time appear to pass quickly.

Recently R. E. Ornstein ${ }^{7}$ has argued against any timesense organ, and he points out that chemical theories cannot cope with those purely psychological manipulations which alter perception of time duration. An interval filled with interesting and complex psychological stimulation is perceived as longer. Ornstein, with many ingenious supporting experiments, proposes that, since perception of duration must be linked with memory, we should use the metaphor of memory storage size to account for perceived duration. Fever, amphetamine, and complexity of stimulus could all be thought to increase the rate at which information is processed in the brain. Consequently the memory units therefrom would occupy more "space" in the memory store, and hence the period from which they arose would be judged as longer.

An important feature of this theory is that the space occupied in the memory store depends also on the coding or " chunking" into storage units. Inefficient chunking would increase storage requirements. Familiar procedures we perform automatically would be chunked as large units, so that when we have driven along a familiar route we may suddenly find we have got to our destination in an apparently short time. When there is very little input to the brain the duration of time will be judged to have been brief. For instance, it has been found that volunteers isolated under conditions of sensory deprivation underestimate the time they have spent and "lose" as much as one day in four. ${ }^{8} 9$

The doctor frequently hears his patients making judgements about time duration, including duration of the delay in falling asleep and duration of total sleep. Since sieep is relatively devoid of information-processing, little storage space should be required for memories arising from it, and in retrospect sleep would tend to be misjudged as short, or even absent, whereas delay in getting to sleep, or intervals of wakefulness while thinking was going on, would use up more storage and so appear to have occupied more of the night. In a recent study of such judgements S. A. Lewis ${ }^{10}$ did indeed find wakefulness overestimated and duration of sleep underestimated, a tendency strikingly enhanced when sleeping-pills were withdrawn.

Poorer capacity to code or chunk incoming information should mean that people of lower intelligence would judge an interval of time to have lasted longer than would people of higher intelligence. And in a recent comparison of Baltimore hospital staff from upper and lower socio-economic groups (and higher and lower intelligence respectively) the lower socio-economic group was found consistently to judge elapsed time as longer. ${ }^{11}$ The authors see the difference as a factor contributing to social conflict and failure of patients in the lower social classes to do well with psychotherapy. At the least it may show that doctors have their priorities wrong if they are more apologetic to middle-class patients kept hanging around in the waiting-room.

1 Hoagland, H., fournal of General Psycholsgy, 1933, 9, 267.

2 Baddeley, A. D., American fournal of Psychology, 1966, 79, 475.

s Aronson, H., Silverstein, A. B., and Klee, G. D., Archives of General Psychiatry, 1959, 1, 469.

- Fischer, R., Griffin, F., and Liss, L., Annals of the New York Academy of Sciences, 1962, 96, 44

" Fischer, R., Annals of the New Y'ork Academy of Sciences, 1967, 138, 440.

Goldstone, S., Boardman, W. K., and Lhamon, W. T., British Fournal of Psychology, 1958, 49, 324

Ornstein, R. E.. On the Experience of Time, 1969. Harmondsworth, Penguin Books.

- Vernon, J A, and McG!ll, T. E., fournal of General Psychology, $1963,69,11$

- Banks, R., and Cappun.. D., Perceptual and Motor Skills, 1962, 14, 74.

10 Lewis, S. A., Britich fournal of Psychology, 1969, 60, 203.

1 Pollock, I. W (khberg, F. M., and Meyer, E., Archives of General Psychiatry 1969, 21, 1 . 